\title{
Análise derivativa de dados hiperespectrais medidos em nível de campo e orbital para caracterizar a composição de águas opticamente complexas na Amazônia
}

\author{
Conrado M. RUDORFF', Evlyn M. L. M. NOVO ${ }^{1}$, Lênio S. GALVÃO ${ }^{1}$, Waterloo PEREIRA FILHO ${ }^{2}$
}

\section{RESUMO}

A técnica de análise derivativa de dados espectrais foi usada para estudar a variação dos constituintes opticamente ativos (COAs) na água, por meio de dados de campo e de imagens do sensor orbital Hyperion/EO-1. A imagem Hyperion usada neste estudo foi adquirida no dia 23 de junho de 2005, no final do período de cheia. Uma campanha de campo foi realizada entre 23 e 29 de junho de 2005, para coletar dados espectrais e limnológicos in situ. A imagem foi pré-processada visando eliminar faixas de pixels anômalos e convertida de valores de radiância para reflectância de superfície, portanto, corrigidos dos efeitos de absorção e espalhamento atmosféricos. Uma análise da correlação foi realizada para examinar a associação da reflectância e de sua primeira derivada espectral com as concentrações dos COAs. Melhores resultados foram obtidos após a diferenciação dos espectros, o que ajudou a reduzir a influência de efeitos indesejáveis, provindos de diferentes fontes de radiância, sobre as medidas de reflectância da superfície da água realizadas em ambos os níveis de aquisição de dados. Por meio de ajustes de regressōes empíricas, considerando o conjunto de dados Hyperion, a primeira derivada espectral em $711 \mathrm{~nm}$ explicou $86 \%$ da variação da concentração de sedimentos inorgânicos em suspensão $\left(\mathrm{mg} \mathrm{l}^{-1}\right)$ e a primeira derivada espectral em $691 \mathrm{~nm}$ explicou $73 \%$ da variação na concentração da clorofila- $a\left(\mu \mathrm{g}^{-1}\right)$. As relaçōes de regressão foram não-lineares, pois, em geral, as águas que se misturam na planície de inundação Amazônica se tornam opticamente complexas. A técnica de análise derivativa hiperespectral demonstrou potenciais para mapear a composição dessas águas.

\section{PALAVRAS-CHAVE}

Sensoriamento remoto hiperespectral; primeira derivada; constituintes opticamente ativos, Hyperion, Amazônia.

\section{Derivative analysis of hyperespectral data measured at field and orbital level to characterize the composition of optically complex waters in the amazon}

\begin{abstract}
Derivative analysis of spectral data was used as a technique to study the variation of optically active constituents (OACs) of water, using field data and hyperspectral imagery of EO-1 Hyperion orbital sensor. The Hyperion image used in this study was acquired on June 23, 2005, at the end of the high water period for the Amazon River. A field campaign was carried out between June 23 and 29, 2005 to collect spectral and limnological in situ data. The image was pre-processed to remove stripes of abnormal pixels and converted from radiance to surface reflectance values, thus, correcting the effects of atmospheric absorption and scattering. A correlation analysis was carried out to examine the association of the spectral reflectance and its first derivative to the concentrations of $O A C$. Better results were obtained after spectra differentiation, which helped to reduce the influence of undesirable effects, coming from different sources of radiance, on the measurements of water surface reflectance taken at both data acquisition levels. Through empirical regression fits, considering the Hyperion dataset, the first spectral derivative at $711 \mathrm{~nm}$ explained $86 \%$ of the variation of suspended inorganic sediment concentration $\left(\mathrm{mg} \mathrm{l}^{1}\right)$, and the first derivative at $691 \mathrm{~nm}$ explained $73 \%$ of the variation of chlorophyll-a concentration $\left(\mu g t^{-1}\right)$. The regression relations were nonlinear because, generally, the water masses that mix in the Amazon floodplain become optically complex. The hyperspectral derivative analysis demonstrated potential for mapping the composition of these waters.
\end{abstract}

KEYWORDS

Hyperspectral remote sensing; first derivative; optically active constituents, Hyperion, Amazon.

1 Instituto Nacional de Pesquisas Espaciais. E-mail: cmr@ltid.inpe.br; evlyn@ltid.inpe.br; lenio@ltid.inpe.br

2 Universidade Federal de Santa Maria. E-mail: waterloo@base.ufsm.br 


\section{INTRODUÇÃo}

O sensoriamento remoto tem contribuído muito para o estudo dos mais diversos ambientes do planeta, ajudando a ampliar a compreensão das estruturas ecossistêmicas e de suas interaçōes. Um dos principais interesses no uso de imagens de satélite em ambientes aquáticos é verificar a variação espacial e temporal da composição da água, possibilitando investigar a origem e o deslocamento de substâncias específicas em suspensão ou dissolvidas na água (Jensen, 2000). Sedimentos em suspensão, pigmentos fotossintetizantes, matéria orgânica dissolvida e as moléculas de água, em si, são os principais agentes que regem as propriedades ópticas inerentes da água e, portanto, são chamados de constituintes opticamente ativos (COAs). Os efeitos espectrais dos COAs sobre a reflectância da água têm sido amplamente discutidos na literatura (Arst, 2003; Bukata, 2000; Kirk, 1994). Este conhecimento científico aliado ao desenvolvimento de sensores, capazes de registrar com maior detalhe as propriedades espectrais dos alvos na superfície terrestre, e de métodos de análise, que permitem a extração de informaçōes cada vez mais específicas e precisas, têm aperfeiçoado o estudo da composição da água por meio de técnicas de sensoriamento remoto (Méléder et al., 2003). No entanto, ainda restam limitações tecnológicas e metodológicas que restringem a obtenção de medidas com precisão satisfatória para águas opticamente complexas em ambientes continentais e costeiros (Arst, 2003). As limitações tecnológicas estão relacionadas às características dos instrumentos em termos de resoluções espaciais, espectrais e radiométricas. As dificuldades metodológicas estão, principalmente, relacionadas às questōes de como isolar a componente da reflectância da água que traz a informação sobre os COAs dos demais fluxos de radiação detectados pelos sensores e como modelar os efeitos de misturas espectrais que ocorrem entre os COAs. As imagens de sensoriamento remoto proporcionam visões sinóticas sobre a área de estudo. Entretanto, é importante destacar que as informaçōes da distribuição espacial de COAs são limitadas apenas à camada mais próxima a superfície da água que, em geral, representa um terço da zona eufótica.

Um sensor imageador hiperespectral tem como vantagem a capacidade de compor uma imagem adquirindo instantaneamente milhares de espectros, com um nível de resolução espectral mais próximo daquele verificado em espectrorradiômetros de campo ou de laboratório. O Hyperion, lançado abordo do satélite Earth Observing One (EO-1) em novembro de 2000, é o primeiro sensor orbital hiperespectral que permite a aquisição de imagens em 242 bandas espectrais (10 $\mathrm{nm}$ de largura cada) posicionadas nas faixas do visível (VIS), do infravermelho próximo (NIR) e do infravermelho de ondas curtas (SWIR). Este sensor não foi projetado para o estudo da água, pois apresenta razão sinal/ruído (SNR) baixa no VIS (Pearlman et al., 2003). No entanto, pesquisas preliminares já demonstraram a sua utilidade no estudo de habitat bentônico
(Kruse, 2003) e no mapeamento da composição de águas costeiras (Vittorio e Dekker, 2003). De fato, ainda há a necessidade de avaliar o desempenho desses dados no estudo de outros ambientes aquáticos como na Amazônia.

Em estudos de águas amazônicas, diferentes técnicas de sensoriamento remoto têm sido utilizadas, a maioria das quais usando modelos de mistura espectral (Adams et al., 1986) aplicados a dados orbitais multiespectrais (Mertes et al., 1993; Novo et al., 2006). Usando imagens Hyperion obtidas em diferentes datas, Rudorff et al. $(2006$; 2007) aplicaram um modelo linear de mistura espectral para explicar a variação espacial da composição de águas amazônicas. Segundo estes autores, esta abordagem apresentou vantagens na análise da dinâmica temporal da água, mas mostrou limitaçôes na estimativa dos COAs.

A técnica de análise da derivada espectral apresenta a vantagem de reproduzir dados hiperespectrais menos sensíveis às componentes de variação que se comportam como constantes aditivas e espectralmente independentes ao longo de uma faixa espectral de alguns nanômetros (Tsai \& Philpot, 1998). Esta possibilidade é importante para estudos do meio aquático natural sujeito aos efeitos de misturas espectrais não-lineares e a condições ambientais de iluminação tão variáveis (Chen et al., 1992; Curran \& Novo, 1988; Han \& Rundquist, 1997; Kondratyev \& Pozdniakov, 1990; Novo et al., 2004). A base matemática da derivada espectral de primeira ordem $\left(d R_{\lambda}\right)$ é estabelecida pela mudança na reflectância $\left(R_{\lambda}\right)$ em função do comprimento de onda ( $\lambda$ ) (Philpot, 1991). Devido à sua natureza - graficamente representada pela inclinação das tangentes em cada ponto da curva-, a derivada evidencia os pontos onde há mudanças bruscas de resposta espectral (Figura 1).

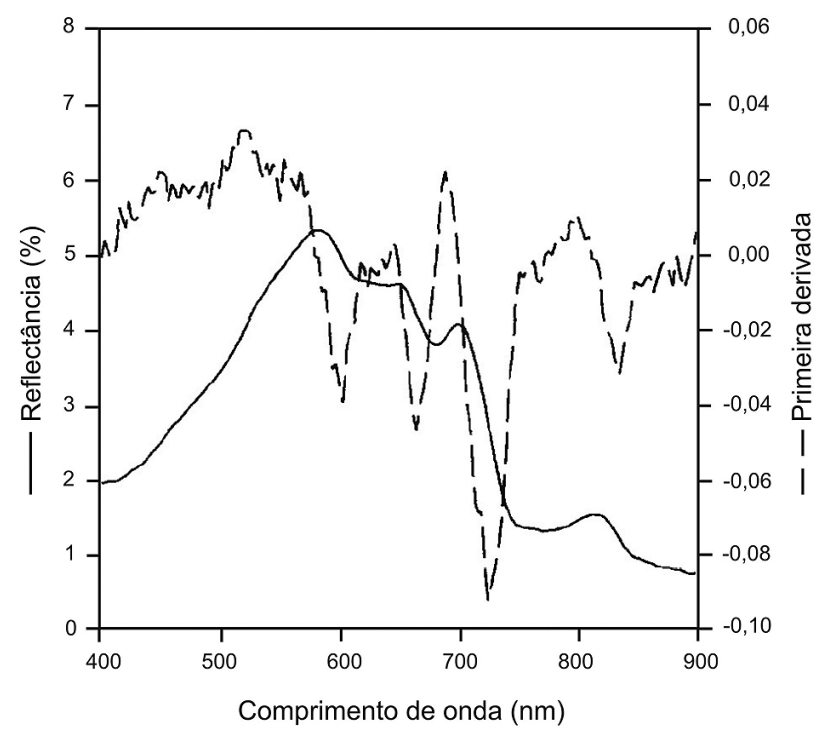

Figura 1 - Exemplo de reflectância e a primeira derivada espectral. (Fonte: Han \& Rundquist, 1997). 
O uso de análise derivativa para estimar a concentração de sedimentos inorgânicos em suspensão foi avaliado por Chen $e t$ al. (1992), que compararam medidas de laboratório e de campo. A reflectância dos sedimentos inorgânicos em suspensão é dominante sobre o comportamento espectral da água. Portanto, em casos de águas turvas, haverá sérias dificuldades em estimar a concentração de clorofila- $a$ usando a reflectância de forma direta, e mesmo considerando a razão de bandas em comprimentos de ondas específicos. Han \& Rundquist (1997) identificaram que a primeira derivada em $691 \mathrm{~nm}$ apresenta a maior correlação com a concentração de clorofila- $a$ e ao mesmo tempo a sua correlação com a turbidez é fraca. Eles, então, apresentaram a primeira derivada espectral como uma alternativa satisfatória para estimar clorofila- $a$ em presença de sedimentos em suspensão.

O objetivo deste trabalho foi verificar o potencial da aplicação da análise derivativa sobre dados hiperespectrais de água, medidos por sensores em nível de campo (Spectron Engineering SE-590) e orbital (Hyperion/EO-1), visando estabelecer relaçôes empíricas para estimar a distribuição espacial de COAs. Os dados foram coletados em uma área de estudo localizada na planície de inundação da Amazônia Central, durante o período de cheia. Relaçōes de regressão entre valores da primeira derivada da reflectância espectral em bandas específicas e concentrações de COAs foram determinadas, após análises comparativas das associações entre ambos os conjuntos de dados (SE-590 e Hyperion), na faixa espectral $450-900 \mathrm{~nm}$, e as medidas dos COAs.

\section{MATERIAL E MÉTODOS}

\section{COLETA DE DADOS ESPECTRAIS E LIMNOLÓGICOS NA ÁREA DE ESTUDO}

A área de estudo localiza-se na região próxima à confluência dos rios Tapajós e Amazonas (Figura 2), sendo formada por uma várzea composta por corpos d'água heterogêneos de diversas origens e composições. No dia 23 de junho de 2005, no final do período de cheia, o sensor Hyperion/EO-1 adquiriu uma imagem sobre o trecho da planície ilustrado na Figura 2. A área alagada contida nesta imagem será referida neste documento como Planície de Inundação Alenquer-Santarém (PIAS).

Foi realizada uma campanha de campo para medir dados espectrais e limnológicos, em 43 estaçôes de coleta (ECs) distribuídas ao longo da PIAS, entre os dias 23 e 29 de junho de 2005. A amostragem de campo foi realizada entre $10 \mathrm{~h} 00 \mathrm{~m} \mathrm{e}$ $14 \mathrm{~h} 00 \mathrm{~m}$ (horário local) para minimizar efeitos de geometria de iluminação durante a aquisição dos dados. O SE-590 foi posicionado sobre cada EC, com orientação de $90^{\circ}$ ou mais de azimute solar e $45^{\circ}$ de inclinação do sensor em relação à vertical e a uma altura de aproximadamente $2 \mathrm{~m}$ acima do nível da água. Em cada EC, foram realizadas seqüencialmente (em menos de 30 s) medidas de radiância da água e da placa de referência de sulfato de bário usando o espectrorradiômetro Spectron Engineering SE-590. O SE-590 mediu a radiância com uma resolução espectral de 2,8 nm ao longo da faixa de 350-1100

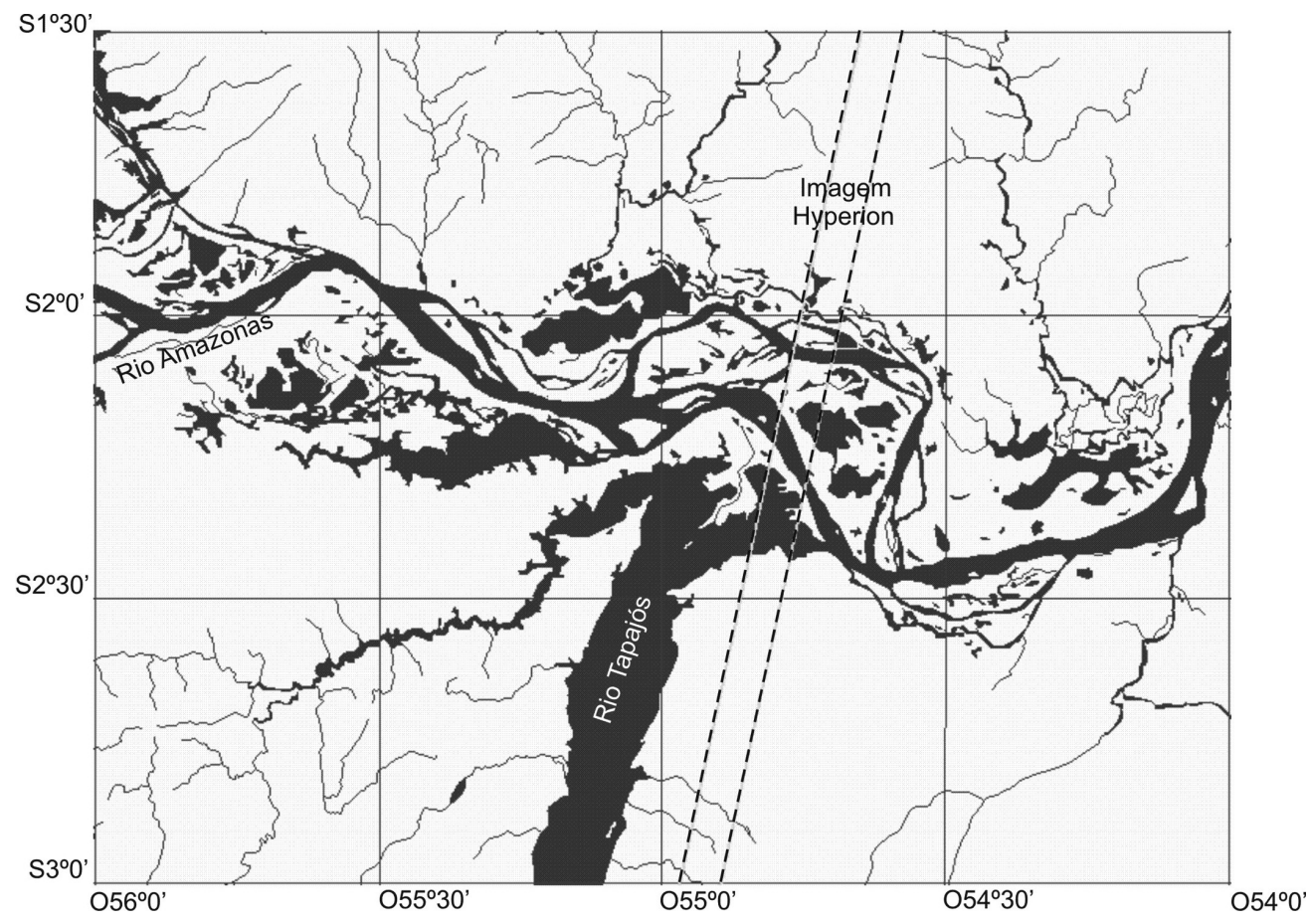

Figura 2 - Localização da área de estudo. As linhas tracejadas delimitam a faixa de aquisição da imagem Hyperion/E0-1. 
nm. O fator de reflectância bidirecional da água $\left(R_{\text {agua }}\right)$ foi calculado usando a seguinte equação:

$$
R_{\text {agua }}(\lambda)=\frac{N D_{\text {agua }}(\lambda)}{N D_{\text {placa }}(\lambda)} \times R_{\text {placa }}(\lambda)
$$

em que $N D_{\text {agua }}$ é o valor de número digital bruto medido sobre a água, $N D_{\text {placa }}$ é o valor de número digital bruto medido sobre a placa de referência, $R_{\text {placa }}$ ó fator de reflectância bidirecional da placa de referência e $\lambda$ representa comprimento de onda.

Imediatamente após a medição espectral, uma amostra de água foi coletada na sub-superfície ( $0,5 \mathrm{~m}$ de profundidade) em garrafa de volume de 5 litros. As amostras de água foram filtradas em campo e conservadas adequadamente por um período de 18 dias, conforme as especificações dos métodos de análise. As concentrações de clorofila- $a(\mathrm{Cl}-a)$ em $\mu \mathrm{g} \cdot \mathrm{l}^{-1}$ (Nush, 1980), sedimentos inorgânicos em suspensão (SIS) em mgl ${ }^{-1}$ (Wetzel e Likens, 1991) e carbono orgânico dissolvido (COD) em ppm (APHA, 1998) foram determinadas em laboratório. As amostras de água foram tomadas na sub-superfície assumindo-se que, desta forma, esteve representada a parte da coluna d'água com maior influência sobre a radiância emergente detectada pelos sensores.

Em cada EC foram, também, tomadas as seguintes medidas complementares: profundidade total do corpo d'água, em metros; transparência (penetração vertical da luz solar na coluna d'água com o disco de Secchi), em metros; turbidez, em NTU (Nephelometric Turbidity Unit), oxigênio dissolvido (OD), em $\mathrm{mgl}^{-1}$, condutividade elétrica (CE), em $\mu \mathrm{S} \cdot \mathrm{cm}^{-1} ; \mathrm{e} \mathrm{pH}$. O sistema de posicionamento Global Positioning System (GPS) foi usado para registrar a localização geográfica.

\section{PRÉ-PROCESSAMENTO DA IMAGEM HYPERION/EO-1}

O pré-processamento da imagem Hyperion envolveu três etapas: correção de pixels ou coluna de pixels com resposta espectral anormal (destriping), correção atmosférica e correção geométrica (registro).

Mesmo após as correçōes radiométricas disponibilizadas para os usuários, ainda restam nas imagens Hyperion pixels ruidosos ou anômalos dispostos seqüencialmente em colunas na forma de "faixas escuras" (stripes) perpendiculares à linha de varredura. Estes ruídos são introduzidos devido a fatores como a nãolinearidade dos detectores, deslocamento da abertura do componente óptico em relação ao plano focal e efeitos de temperatura. Para a correção dos mesmos, foi utilizada a metodologia proposta por Goodenough et al. (2003) e Han et al. (2002), sendo substituída a resposta radiométrica dos pixels nas faixas ruidosas pela média de seus vizinhos na direção horizontal.

Para corrigir os efeitos atmosféricos sobre a imagem Hyperion, foi utilizado o aplicativo Atmosphere Correction Now (ACORN) (ImSpec, 2001). O algoritmo se baseia no código MODTRAN-
4 para minimizar os efeitos de espalhamento e absorção dos gases da atmosfera. Dentre as 242 bandas disponíveis, apenas aquelas radiometricamente calibradas e que se encontravam fora da faixa espectral de intensa interferência pelo vapor d'água na atmosfera (em torno de 1400 e $1900 \mathrm{~nm}$ ) foram utilizadas para fins de correção atmosférica ( 157 bandas). O algoritmo requer a inserção dos seguintes parâmetros: altitude do sensor; data e hora de aquisição; localização (latitude/longitude, elevação média); e visibilidade da atmosfera. O cálculo do vapor d'água é feito pixela-pixel usando os dados da própria imagem ou mais especificamente das bandas de absorção posicionadas em $940 \mathrm{e}$ $1140 \mathrm{~nm}$.

Após as etapas de destriping e correção atmosférica, foi realizada a correção geométrica dos dados aplicando um ajuste polinomial de segunda ordem a um conjunto de pontos de controle de terreno, que foram distribuídos na imagem Hyperion e em uma imagem Thematic Mapper do satélite Landsat-5 orto-retificada, a qual serviu como referência. O modelo polinomial ajustado foi usado para reamostrar os pixels da imagem Hyperion pelo método de vizinho mais próximo.

\section{ANÁLISE DOS DADOS}

Em cada etapa das análises que serão descritas a seguir, os espectros de reflectância de campo obtidos com o SE-590 foram comparados, quanto à similaridade espectral, com os espectros Hyperion (média de 4 x 4 pixels) extraídos de pixels da cena geograficamente correspondentes aos locais das ECs.

Como passo preparatório ao cálculo das derivadas, um filtro de média móvel de três pontos (bandas) foi aplicado sobre os espectros de reflectância para evitar que ruídos fossem amplificados durante a diferenciação (Tsai \& Philpot, 1998). Um filtro de média móvel simples assume o valor espectral médio de todos os pontos dentro de um intervalo espectral específico, como sendo o novo valor do ponto central deste intervalo. Assim, o novo valor de reflectância $(\widetilde{R})$ para o $i$-ésimo elemento pode ser expresso pela equação:

$$
\widetilde{R}_{i}=\frac{\sum_{j=i-l}^{i+l} R_{j}}{N}
$$

em que $l$ é o número de elementos anteriores e posteriores ao elemento corrente $i$; e $N$ (número de pontos do intervalo) é o tamanho do filtro (2l+1). Conforme esperado, os espectros de campo medidos com melhor resolução espectral pelo SE-590 mostraram pequenas diferenças após a filtragem. Por outro lado, a aplicação do filtro nos espectros Hyperion reduziu algumas oscilaçôes radiométricas introduzidas pela baixa razão sinal/ruído deste sensor. 
A primeira derivada $\left(d R_{\lambda}\right)$ do espectro de reflectância com relação ao comprimento de onda $x$ em um determinado ponto $i$ pôde ser numericamente aproximada por um esquema de diferenças finitas usando a aproximação central ou simétrica, expresso pela equação:

$$
\frac{d R_{\lambda}}{d x} \cong \frac{R_{i+1}-R_{i-1}}{2 \Delta x}
$$

em que $\Delta x$ é a separação entre duas bandas sucessivas. Sendo $\Delta x$ $=x_{j}-x_{k}$, considera-se que $x_{j}>x_{k}$ e que o intervalo entre as bandas seja constante.

A análise derivativa foi conduzida visando identificar as variáveis espectrais que permitissem estimar a distribuição espacial da concentração dos COAs, por meio de regressôes empíricas, e mapeá-las sobre a imagem Hyperion. Uma máscara foi usada para distinguir as massas de água sobre a cena da imagem Hyperion para facilitar o mapeamento. Um simples limiar de $1 \%$ de reflectância na banda de $2300 \mathrm{~nm}$ foi utilizado para gerar esta máscara, uma vez que, na faixa espectral do infravermelho de ondas curtas (SWIR) a água absorve praticamente toda radiação incidente, enquanto os demais componentes de cena apresentam reflectância mais elevada. A máscara formada se constituiu em uma imagem binária na qual os pixels sobre corpos d'água apresentaram valor 1 e os pixels sobre alvos terrestres e vegetação aquática apresentam valor 0 .

\section{RESULTADOS E DISCUSSÃO}

Para ilustrar os efeitos espectrais da reflectância e sua primeira derivada em resposta às variaçóes de concentração dos $\mathrm{COAs}$, tanto em nível de campo como orbital, foram selecionadas amostras de quatro ECs, que representam os principais ambientes aquáticos lóticos e lênticos da PIAS. A Tabela 1 apresenta as medidas limnológicas obtidas nestas ECs, cujas posições podem ser observadas na Figura 9a.

O Amazonas é o rio principal da planície de inundação. Durante o seu percurso dos Andes até o oceano, o conteúdo de sólidos nele dissolvidos decai significativamente devido à mistura das massas de água dos seus tributários, que geralmente são mais pobres em sólidos dissolvidos (Furch \& Junk, 1997). Mesmo assim altas concentraçōes de sólidos dissolvidos ocorrem nas águas do rio Amazonas que cruzam a PIAS. Tal observação é sustentada pela CE medida na EC-30 $\left(51,40 \mu \mathrm{S} \cdot \mathrm{cm}^{-1}\right)$ (Tabela 1). A concentração de SIS no rio também se mostrou tipicamente elevada $\left(64,33 \mathrm{mgl}^{-1}\right)$.

Quando as águas do rio Amazonas entram na planície de inundação durante a cheia, os sedimentos em suspensão são decantados e a turbidez diminui significativamente. A concentração de SIS medida na EC-17, localizada na várzea Boca do Surubim-açú a $2 \mathrm{~km}$ do rio Amazonas, foi de 5,34 mg. $\mathrm{l}^{1}$
Tabela 1 - Constituintes opticamente ativos (COAs) da água e parâmetros limnológicos obtidos em 4 estações de coleta (EC). SIS - sedimentos inorgânicos em suspensão, $\mathrm{Cl}$-a - clorofila-a, COD - carbono orgânico dissolvido, NTU - unidade nefelométrica de turbidez, OD - oxigênio dissolvido, CE - condutividade elétrica.

\begin{tabular}{ccccc}
\hline Local & Rio Amazonas & $\begin{array}{c}\text { Boca do Surubim- } \\
\text { Açu }\end{array}$ & Lago Curumu & Rio Tapajós \\
\hline Amostra & EC-30 & EC-17 & EC-03 & EC-40 \\
\hline SIS $(\mathrm{mg} / \mathrm{l})$ & 64,33 & 5,34 & 1,20 & 0,35 \\
\hline Cl-a $(\mu \mathrm{g} / \mathrm{l})$ & 2,08 & 3,78 & 31,00 & 3,36 \\
COD $(\mathrm{ppm})$ & 5,23 & 4,83 & 5,16 & 2,09 \\
\hline Turbidez $(\mathrm{NTU})$ & 66,10 & 22,30 & 6,30 & 2,40 \\
\hline Transparência $(\mathrm{m})$ & 0,30 & 0,70 & 1,10 & 2,90 \\
\hline Profundidade $(\mathrm{m})$ & 23,50 & 4,60 & 5,00 & 17,00 \\
\hline OD $(\mathrm{mg} / \mathrm{l})$ & 2,96 & 3,26 & 6,30 & 6,27 \\
\hline CE $(\mu \mathrm{S} / \mathrm{cm})$ & 51,40 & 51,00 & 72,80 & 18,20 \\
\hline pH & 6,39 & 6,42 & 6,66 & 6,78 \\
\hline
\end{tabular}

(Tabela 1). Além de menores valores de turbidez, as águas dos lagos durante o período de cheia contêm maior fração orgânica nos sedimentos em suspensão, principalmente, em função dos detritos de animais e plantas que se desenvolveram durante a fase terrestre antecedente (Furch \& Junk, 1997).

O lago Curumu recebe contribuição de águas do rio Amazonas ao sul e de águas que drenam a terra-firme ao norte. As concentraçōes de sólidos dissolvidos e nutrientes em igarapés de terra firme são geralmente menores em relação ao rio Amazonas (Forsberg et al., 1988). No entanto, águas que drenam áreas antropizadas de terra-firme recebem grande quantidade de sólidos dissolvidos e nutrientes (Biggs et al. 2004). Portanto, as maiores concentrações de $\mathrm{Cl}-a$ observadas na EC-03 (Tabela 1), talvez possam ser explicadas pela baixa turbidez do lago aliada à contribuição de nutrientes, principalmente, por desflorestamento e atividade de criação de gado na região.

O rio Tapajós (EC 40) é um grande tributário do rio Amazonas. As suas águas são claras, apresentando baixas concentraçôes de sedimentos em suspensão e matéria orgânica dissolvida (Tabela 1). A profundidade da zona eufótica na água clara do rio Tapajós é elevada, porém a produtividade fitoplanctônica medida foi baixa $\left(3,36 \mu \mathrm{gl}^{-1}\right)$, pois essas águas são relativamente pobres em nutrientes durante o período que corresponde à cheia do rio Amazonas.

\section{RELAÇÕES ENTRE A REFLECTÂNCIA E CONSTITUINTES OPTICAMENTE ATIVOS DAS ÁGUAS}

A Figura 3 apresenta exemplos de espectros de reflectância $\left(R_{\lambda}\right)$ medidos em campo (SE-590) e em nível orbital (Hyperion), referentes as quatro ECs apresentadas na Tabela 1 . Foi notada uma grande semelhança entre os espectros medidos por ambos os sensores nas estaçōes EC-40, EC-17 e EC-30, tanto em termos das feições espectrais quanto em termos de albedo (reflectância 
média na faixa 450-900 nm), conforme as suas respectivas concentrações de COAs. A estação EC-03 (Figura 3) foi a que apresentou maior variação de reflectância entre os dois níveis de aquisição. De fato, existem diversos fatores que afetam as medidas de maneira distinta de acordo com o nível de aquisição de dados (Milton, 1987; Chen et al., 1992; Curran et al., 1987). Em nível orbital, apesar dos espectros serem medidos instantaneamente, eles estão sujeitos, principalmente, às incertezas oriundas do modelo de correção atmosférica (Kaufman, 1987; 1989). Em nível de campo, as incertezas podem ser decorrentes de fatores como: medidas em dias e horários diferentes; variações na irradiância e na geometria de iluminação solar durante a coleta de dados; e reflectância especular. A movimentação da superfície da água provocada pela ação do vento intensifica os efeitos da reflectância especular.

A estação EC-40, localizada no rio Tapajós, apresenta medidas de uma água clara com baixas concentrações de COAs; conseqüentemente, a sua reflectância espectral na faixa do VIS
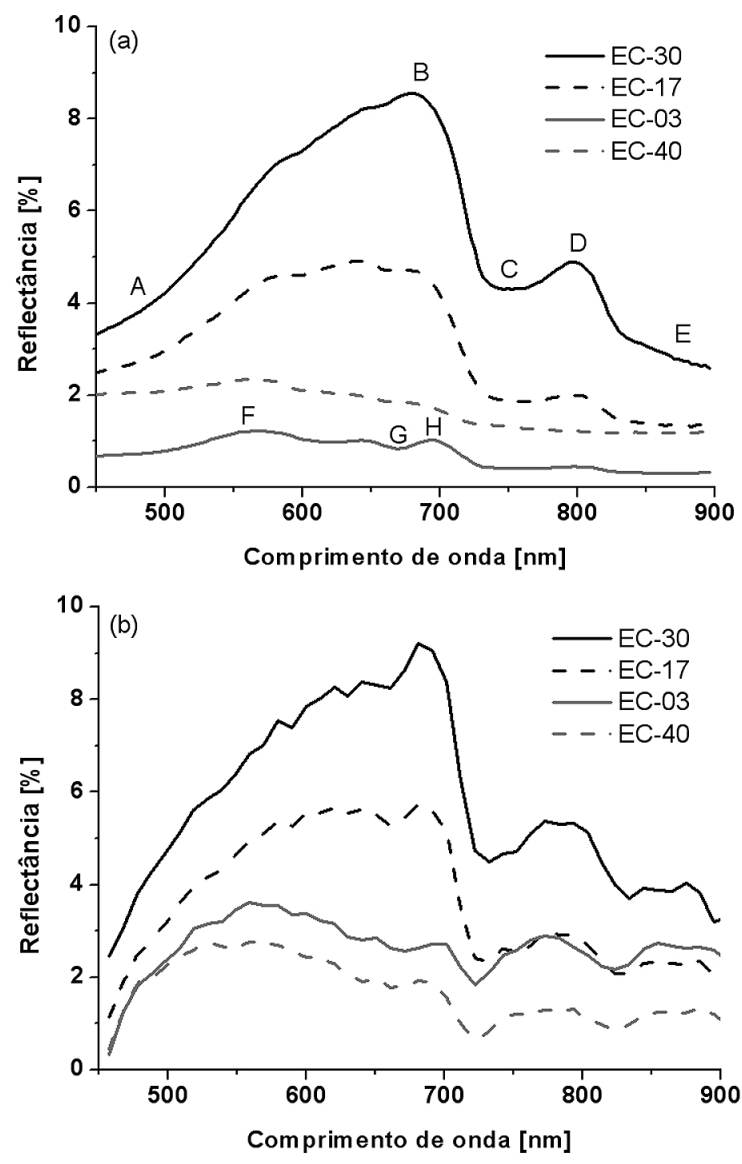

Figura 3 - (a) Exemplos de espectros de reflectância de água medidos pelo SE-590 nas estações de coleta durante a campanha de campo realizada entre 23/06/05 e 29/06/05; (b) Espectros extraídos de pixels da imagem Hyperion/EO-1 adquirida em 23/06/2005 para locais aproximadamente correspondentes às estações de coleta de campo. ao NIR é baixa e moldada principalmente pelas propriedades ópticas das moléculas de água pura, que produzem um aumento exponencial de espalhamento em direção aos comprimentos de onda mais curtos e de absorção em direção aos comprimentos de onda mais longos. A propriedade não-lambertiana da superfície da água, que produz reflectância especular, não varia para os diferentes tipos de água. No entanto, por se tratar de um efeito aditivo, foi verificado que este tipo de interferência se torna mais expressivo sobre as medidas de baixa reflectância que ocorrem nas águas de baixa turbidez.

A matéria orgânica dissolvida absorve radiação na faixa de comprimentos de onda do azul e pode gerar espalhamento por fluorescência no NIR, porém ambos os efeitos produzem feições espectrais com sinais baixos de reflectância e pouco notáveis. Portanto, em geral, é extremamente difícil detectar variações de concentração de matéria orgânica dissolvida por meio de dados de reflectância, uma vez que as respostas espectrais produzidas pelos demais COAs são bem mais dominantes.

As concentrações de $\mathrm{Cl}$ - $a$ são relativamente baixas no período de cheia (Tabela 1) e, portanto, seus efeitos espectrais são pouco expressivos. Apesar disto, três feições espectrais características associadas à clorofila das algas na água foram evidentes nos espectros das estaçóes EC-40 e EC-03, em ambos os conjuntos de dados (Figura 3): (1) reflectância de biomassa algal em torno de 560 nm (F) (Dekker et al., 1991); (2) um mínimo de reflectância em $670 \mathrm{~nm}(\mathrm{G})$, causado por absorção da clorofila; e (3) um máximo de reflectância proeminente em torno de 705 $\mathrm{nm}(\mathrm{H})$, devido ao efeito de fluorescência de clorofila induzida pelo Sol (Dekker et al., 1991; Gitelson, 1992).

$\mathrm{O}$ aumento da concentração de sedimentos em suspensão provoca aumento de reflectância em toda a faixa espectral estudada (Chen et al., 1992; Curran \& Novo, 1988). Nota-se na curva da estação EC-30, localizada no rio Amazonas, faixas de absorção de radiação em torno de: $450 \mathrm{~nm}(\mathrm{~A})$, devido às propriedades espectrais conjuntas dos sedimentos em suspensão e da matéria orgânica dissolvida; $750 \mathrm{~nm}(\mathrm{C})$, por absorção pela rotação da molécula de água; e $900 \mathrm{~nm}$ (E), igualmente pela água. Devido ao efeito destas faixas de absorção e ao espalhamento produzido pelas partículas do sedimento, dois picos de reflectância se tornam proeminentes em $690 \mathrm{~nm}$ (B) e $800 \mathrm{~nm}$ (D).

Uma analise de correlação ( $n=43$ estações de campo) foi aplicada às bandas compreendidas na faixa de comprimento de onda entre 450 e $900 \mathrm{~nm}$ para examinar a associação entre a reflectância espectral e os COAs. De acordo com a Figura 4, o formato geral dos correlogramas de cada variável foi similar entre os dois níveis de aquisição de dados (SE-590 e Hyperion), indicando que ambos os sensores foram sensíveis às variaçōes dos COAs na água. Comparativamente, o conjunto de dados Hyperion (Figura 4b) apresentou correlações ligeiramente maiores do que o conjunto de dados SE-590 (Figura 4a). Isto significa que, mesmo considerando as incertezas de correção 
atmosférica, os dados orbitais aparentemente apresentaram menores interferências dos fatores mencionados sobre a reflectância do que as medições em condiçôes de campo. $\mathrm{O}$ fato de que a imagem Hyperion adquirida instantaneamente apresentou boa associação com as medidas de COAs realizadas em campo, em um intervalo de tempo de cinco dias, também revela que uma condição de relativa estabilidade na composição da água foi verificada, fato característico do período de cheia (Barbosa, 2005).

A Figura 4 mostra que as correlações entre os valores de medidas de reflectância e SIS foram positivas em toda a faixa espectral. Para os dados Hyperion, o valor máximo de $r$ foi de $+0,79$, observado na banda $690 \mathrm{~nm}$ (Figura 4b). Por outro lado, as correlações entre reflectância e $\mathrm{Cl}-a$ foram baixas; de fato, os valores absolutos de $r$ foram menores que 0,36 . Estes resultados sugerem que os demais COAs, particularmente SIS e detritos, dominaram a composição do sinal de reflectância espectral no período estudado. Portanto, torna-se difícil estimar a concentração
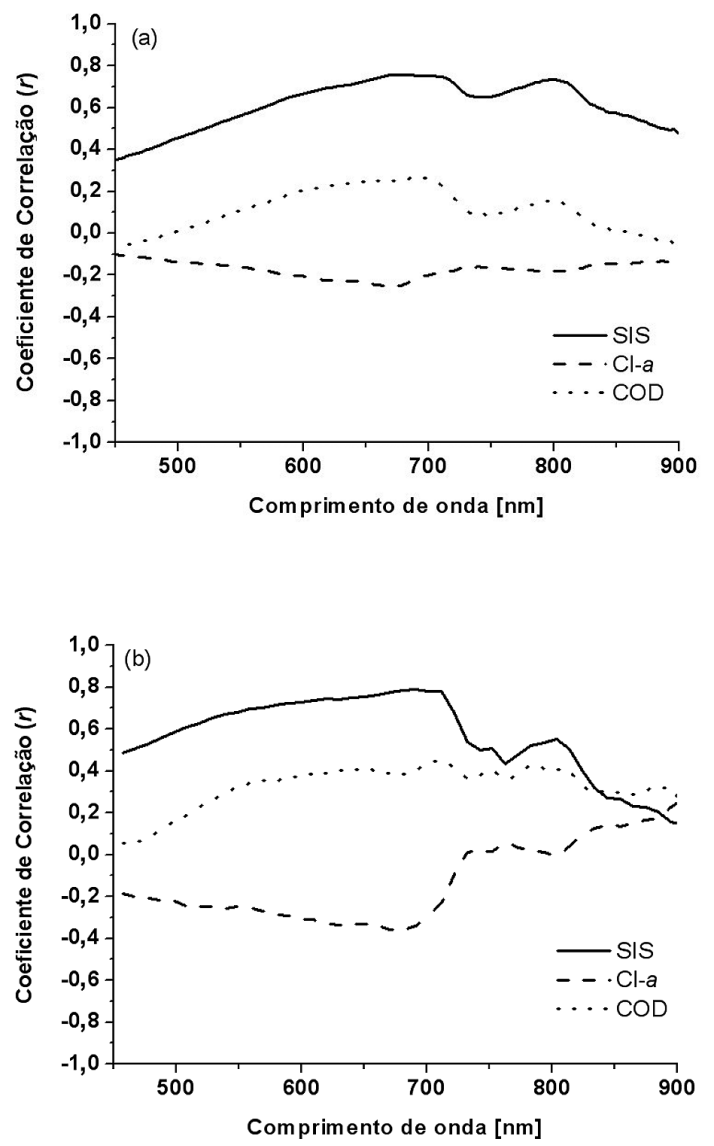

Figura 4 - Variações da correlação entre a reflectância e os constituintes opticamente ativos da água para dados de (a) campo (SE-590) e (b) orbital (Hyperion). de clorofila- $a$ neste período usando dados de reflectância e a diferenciação destes dados (análise derivativa) pode ser uma alternativa para tal finalidade, como será discutido a seguir.

A medida de carbono orgânico dissolvido (COD) é considerada uma boa indicadora de matéria orgânica dissolvida, por diversos autores (p.ex., Rochelle-Newall e Fisher, 2002). No entanto, conforme esperado, as correlações entre COD e reflectância também foram baixas para ambos os níveis de aquisiçāo de dados (Figura 4). As águas escoadas pelo rio Amazonas apresentam elevadas concentraçôes de sedimentos em suspensão e matéria orgânica dissolvida. Conseqüentemente, o correlograma referente ao COD foi predominantemente positivo e acompanhou a forma do correlograma referente ao SIS, por este COA apresentar maior dominância sobre a reflectância espectral. Assim, os conjuntos de dados espectrais não apresentaram qualquer potencial preditivo para a variável COD.

As medidas de profundidade, turbidez e transparência foram adotadas como variáveis auxiliares às medidas dos COAs. Os sedimentos em suspensão foram os principais agentes que determinaram o aumento da turbidez e a redução da transparência. Foi observada uma alta correlação entre a reflectância e a turbidez (p. ex., $r=+0,91$; em $690 \mathrm{~nm}$ ) e uma correlação negativa entre a reflectância e a transparência (p. ex., $r$ $=-0,78 ; \mathrm{em} 701 \mathrm{~nm}$ ) com os dados Hyperion. A profundidade dos corpos d'água não apresentou correlação significativa com a reflectância ao longo da faixa de comprimento de onda analisada. Isto indica que, de maneira geral, não houve forte interferência da radiância refletida pelo material do fundo dos corpos d'águas sobre as medidas de reflectância da água. Esta informação também indica que durante o período de cheia, em que foi adquirida a imagem Hyperion, não foi verificada influência da variação em profundidade sobre a ressuspensão de sedimentos de fundo pela ação do vento, comum em ambientes rasos e abertos. Entre os fatores que contribuíram para que não fosse verificada uma relação entre a profundidade dos corpos d'água e a medida de reflectância podemos citar: a profundidade média dos corpos d'água de $6 \mathrm{~m}$; a profundidade média da zona eufótica de $3 \mathrm{~m}$; e a turbidez média das massas de água de 20 NTU.

\section{RELAÇÕES ENTRE A PRIMEIRA DERIVADA E CONSTITUINTES OPTICAMENTE ATIVOS DA ÁGUA}

A Figura 5 apresenta as curvas obtidas após diferenciação dos espectros de reflectância medidos nas quatro ECs (Tabela 1). A primeira derivada espectral $\left(d R_{\lambda}\right)$ aplicada sobre a reflectância reduziu variaçōes de constantes aditivas de radiação em ambos os níveis de aquisição de dados, realçando determinadas feições espectrais. Ao contrário da diferença de reflectância observada nos dados de reflectância entre EC-03 e EC40 (Figura 3), ambas as águas de baixa turbidez, as curvas espectrais dessas estações mostraram similaridade após a diferenciação. A principal diferença entre elas foi observada em torno de $690 \mathrm{~nm}$, por um pico mais 
pronunciado na curva EC-03, devido a maior concentração de $\mathrm{Cl}-a$ (Figura 5). As principais feiçôes prontamente observadas entre as derivadas espectrais EC-30 e EC-17 são os valores espectrais mínimos em $710 \mathrm{~nm}$ e $820 \mathrm{~nm}$, correspondentes ao aumento da concentração de SIS.

As correlaçôes entre a primeira derivada e as concentrações de SIS e Cl- $a$ foram calculadas ( $n=30$ estaçôes de campo). Os correlogramas obtidos apresentaram resultados similares em ambos os níveis de aquisição de dados (Figura 6). Os resultados para o SE-590 apresentaram valores de $r$ em torno de $+0,75$ para a relação entre SIS e $d R$, ao longo da faixa de comprimento de onda de 455-630 nm (Figura 6a). Nesta faixa espectral, apenas as bandas individuais em torno de $569 \mathrm{~nm}, 610 \mathrm{~nm}$ e $640 \mathrm{~nm}$ atingiram correlaçōes próximas a 0,75 para os dados Hyperion (Figura 6b). Correlaçôes negativas entre SIS e $d R_{\lambda}$ maiores que 0,80 foram observadas para os dados SE- 590 , ao longo da faixa de comprimento de onda de 710-740 nm e 805-855 nm. Para dados Hyperion foram observadas correlaçōes de $-0,65$ e - 0,75 nas faixas espectrais de 701-732 nm e 813-834 nm, respectivamente.

O coeficiente máximo de correlação entre SIS e $R_{\lambda}$ foi de $+0,79 \mathrm{em} 681 \mathrm{~nm}$ (Figura 4b), enquanto uma maior correlação
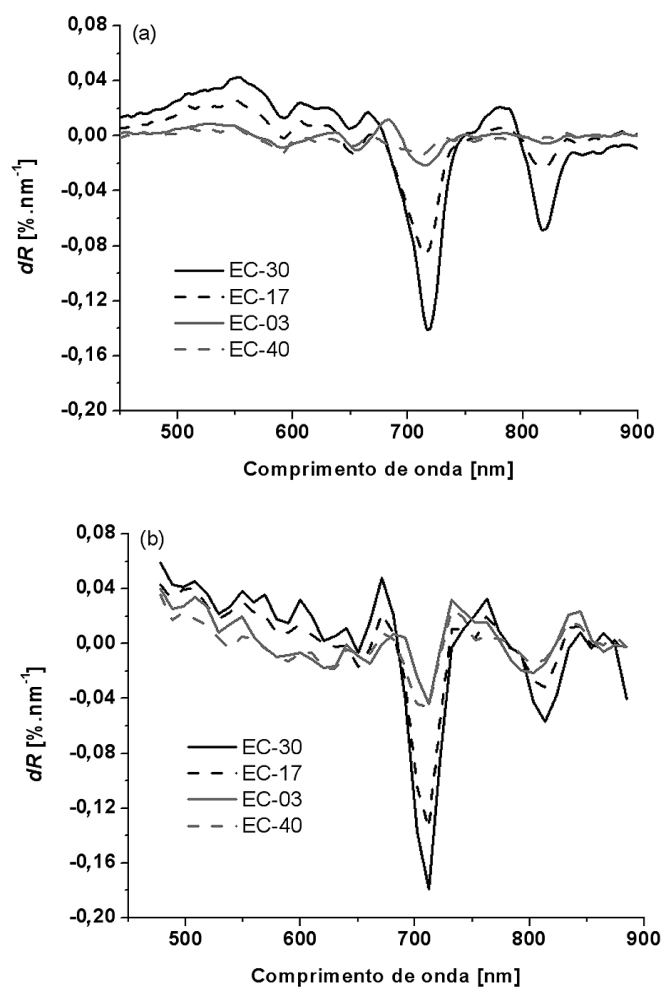

Figura 5 - Exemplos de espectros da água resultantes da aplicação da primeira derivada $\left(d R_{\lambda}\right)$ sobre dados de reflectância de (a) campo (SE-590) e (b) orbital (Hyperion). entre SIS e $d R_{\lambda}(r=+0,85)$ foi obtida em $671 \mathrm{~nm}$ para os dados Hyperion (Figura 6b). Em ambos os conjuntos de dados (SE590 e Hyperion), os valores absolutos de correlação entre $\mathrm{Cl}-a \mathrm{e}$ $R_{\lambda}$ foram menores que 0,36 (Figura 4). Após a diferenciação dos espectros de reflectância, a relação entre $\mathrm{Cl}-a$ e $d R_{\lambda}$ apresentou um valor de correlação de $+0,8$ em torno de $691 \mathrm{~nm}$ para os dados SE-590 (Figura 6a) e de $+0,7$ para os dados Hyperion (Figura 6b). Nota-se nos gráficos da Figura 6, que em torno da banda $691 \mathrm{~nm}$ foram observados valores negativos de $r$ entre $d R_{\lambda}$ e SIS (p.ex., $\mathrm{r}=-0,57$; dados Hyperion). Portanto, em concordância com Han \& Rundquist (1997), pôde-se concluir que a primeira derivada espectral em $691 \mathrm{~nm}\left(d R_{691}\right)$, gerada pela diferença espectral entre a absorção em $670 \mathrm{~nm}$ seguida de espalhamento por fluorescência em $705 \mathrm{~nm}$, é útil para estimar clorofila- $a$ na presença de outros COAs.

Os dados de reflectância Hyperion apresentaram maiores valores absolutos de $r$ do que os dados do SE-590 (Figura 4). Após a aplicação da primeira derivada, os dados SE-590 passaram a se correlacionar melhor com as concentrações de SIS e $\mathrm{Cl}-a \mathrm{em}$ comparação com os dados Hyperion (Figura 6). Supõe-se, portanto, que a primeira derivada espectral tenha sido mais eficiente na redução da variabilidade espectral introduzida pelas
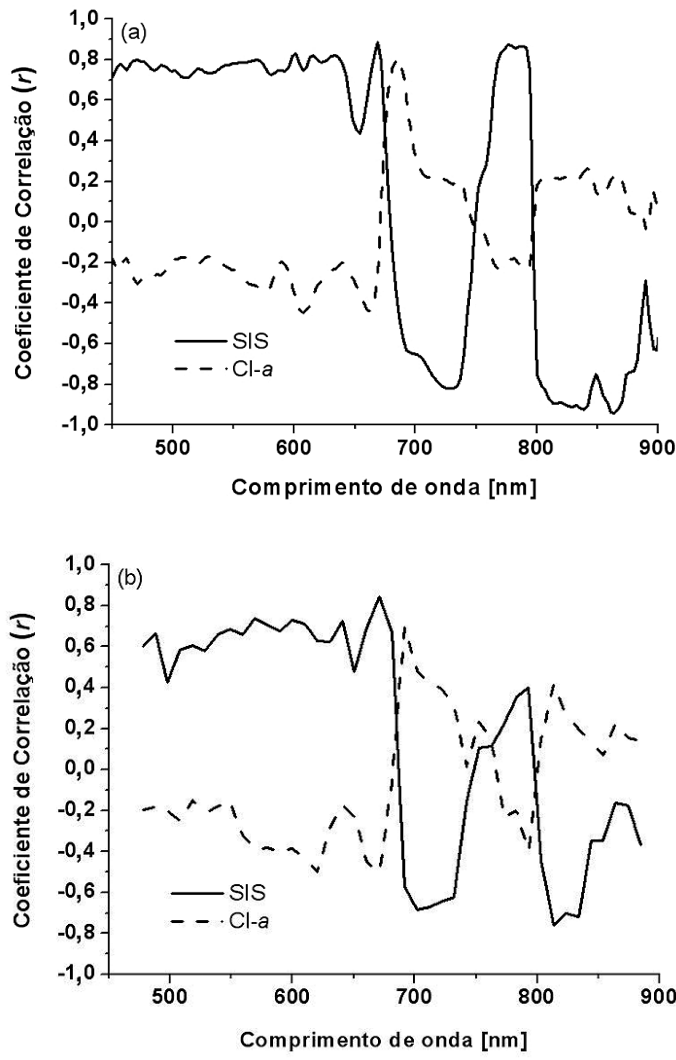

Figura 6 - Variações da correlação entre a primeira derivada espectral e os constituintes opticamente ativos da água para dados de (a) campo (SE590) e (b) orbital (Hyperion). 
incertezas sobre as medidas de campo do que sobre as medidas orbitais.

\section{REGRESSÕES EMPÍRICAS PARA INFERÊNCIA DA COMPOSIÇÃO DA ÁGUA POR MEIO DA IMAGEM HYPERION}

As bandas do sensor Hyperion, cujos valores da primeira derivada espectral $\left(d R_{\lambda}\right)$ apresentaram os melhores resultados de correlação na Figura $6 \mathrm{~b}$, foram testadas para estimar na imagem a distribuição das concentrações de sedimentos inorgânicos em suspensão (SIS) e clorofila- $a(\mathrm{Cl}-a)$ nos corpos d'água. A opção pela transformação da variável dependente e por ajuste polinomial foi considerada a partir do critério de priorizar o aumento de $\mathrm{R}^{2}$, para oferecer maior potencial de predição às regressōes (Neter $e t$ al., 1996). Dentre as $43 \mathrm{ECs}$ amostrais, 30 amostras aleatórias foram utilizadas para o estabelecimento das regressōes e 13 amostras foram separadas para testar suas exatidóes.

A Figura 7a apresenta o modelo empírico usando a primeira derivada em $711 \mathrm{~nm}\left(d R_{711}\right)$ para mapear a concentração de SIS em mg. $1^{-1}$ sobre a PIAS. A regressão ajustada entre $\log \left(\right.$ SIS) e $d R_{711}$ apresentou um coeficiente de determinação $\left(\mathrm{R}^{2}\right)$ de 0,86 . Quando aplicado ao conjunto de amostras separadas para o teste de exatidão, o modelo resultou em um erro quadrático médio

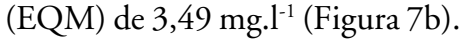

O modelo empírico usando a primeira derivada em $691 \mathrm{~nm}$ $\left(d R_{69}\right)$ para estimar a concentração de $\mathrm{Cl}-a \mathrm{em} \mu \mathrm{gl}^{-1}$ é apresentado
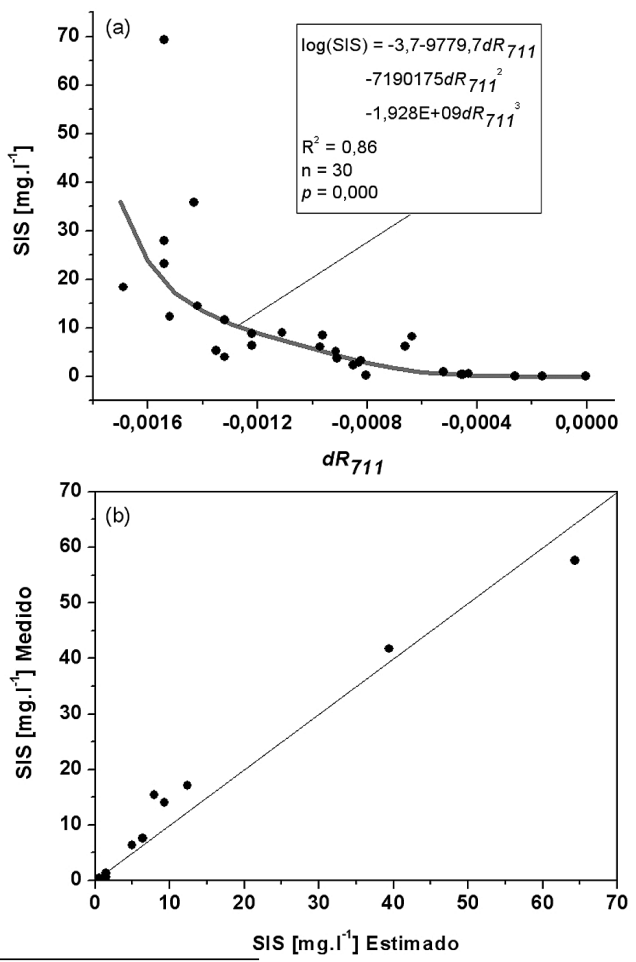

Figura 7 - (a) Modelo empírico para estimar a concentração de sedimentos inorgânicos em suspensão (SIS) no período de cheia a partir de $d R_{711}$; (b) Teste de exatidão do modelo. na Figura 8 a com $R^{2}$ igual a 0,73 . Este comprimento de onda também foi utilizado por Han \& Rundquist (1997) para estimar $\mathrm{Cl}-a$ em águas turvas. Quando aplicado ao conjunto de amostras separadas para o teste de exatidão, o modelo produziu um EQM de $3,97 \mu \mathrm{gl}^{-1}$ (Figura 8b).

A Figura 9a mostra uma composição colorida normal da área de estudo obtida com as bandas do sensor Hyperion posicionadas em $640 \mathrm{~nm}$ (vermelho), $549 \mathrm{~nm}$ (verde) e $457 \mathrm{~nm}$ (azul). As Figuras 9c e $9 \mathrm{~b}$ apresentam os resultados obtidos da aplicação dos modelos empíricos das Figuras 7 e 8 para estimar a concentração em corpos d'água de SIS e $\mathrm{Cl}-a$, respectivamente. O mapeamento da variação espacial da concentração de SIS (Figura 9c) evidenciou baixas concentraçōes no rio Tapajós e elevadas concentraçôes no rio Amazonas, mostrando a gradativa sedimentação de sedimentos fluviais devido à redução da correnteza nos lagos (Fisher \& Parsley, 1979; Forsberg, 1988; Melack \& Forsberg, 2004; Mertes, 1994; Mertes et al., 1996). $\mathrm{O}$ mapeamento da variação espacial da concentração de $\mathrm{Cl}-a$ (Figura 9b) indicou baixas concentraçōes sobre o rio Tapajós por limitaçōes de nutrientes e sobre o rio Amazonas por limitaçōes de luz. Sobre os lagos da várzea de águas menos turva (maior profundidade eufótica), mas que recebem contribuiçôes de águas do rio Amazonas (ricas em nutrientes), foi observado o aumento de fitoplâncton. As concentrações de fitoplâncton aumentam em direção às margens dos lagos. A exposição do fundo no período
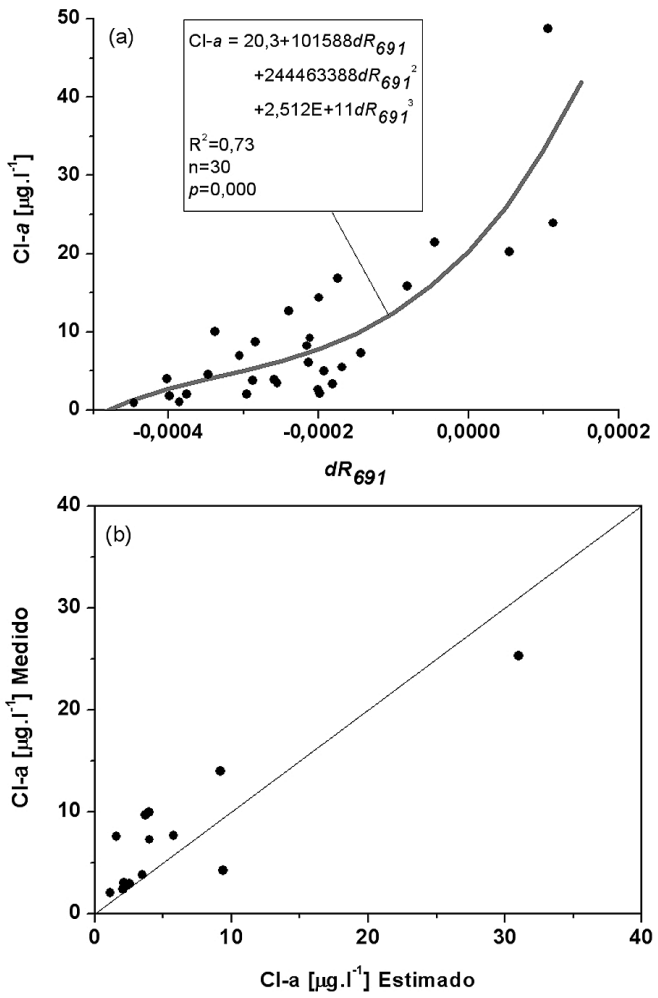

Figura 8 - (a) Modelo empírico para estimar a concentração de clorofila-a (Cl-a) no período de cheia a partir de $d R_{691}$; (b) Teste de exatidão do modelo. 
de água baixa faz com que os nutrientes adsorvidos pelos sedimentos se tornem disponíveis para o crescimento de plantas (fase terrestre). Estes nutrientes são subseqüentemente liberados na água durante o período de cheia quando as plantas se decompõem (fase aquática) (Furch \& Junk, 1997). A maior produção de fitoplâncton ocorrida no período da aquisição da imagem foi verificada nas águas ao norte do lago Curumu (Figura 9b). Este fato pode ser explicado por este lago apresentar água com baixa turbidez durante o período de cheia e receber contribuições de nutrientes de águas que drenam regiōes de terrafirme, onde são observadas atividades conhecidas por causar poluição da água (p.ex., desflorestamento e criação de gado) (Biggs et al., 2004).

\section{CONCLUSÕES}

A análise espectral conduzida para construir relações empíricas indicou uma maior eficiência da primeira derivada espectral para estimar a distribuição espacial da concentração de sedimentos inorgânicos em suspensão e de clorofila- $a$ em relação ao uso de valores originais de reflectância. Diferentemente das regressões lineares obtidas por Chen et al. (1992) e Han \& Rundquist (1997), os ajustes das regressóes entre os dados Hyperion e as concentraçôes de sedimentos inorgânicos em suspensão e de clorofila- $a$ foram não-lineares. Isto aponta para a maior complexidade óptica das águas continentais da planície de inundação amazônica.

Apesar das diferenças entre os níveis de aquisição e as características dos sensores, houve grande coerência entre os resultados obtidos com o Hyperion/EO-1 e o SE-590, indicando que o sensoriamento remoto hiperespectral é uma ferramenta auxiliar importante para o estudo da composição da água na planície amazônica. Neste trabalho, as informações extraídas da imagem hiperespectral adquirida pelo sensor orbital Hyperion/ EO-1 permitiram mapear as concentraçōes de sedimentos inorgânicos em suspensão e clorofila-a, sobre os sistemas aquáticos heterogêneos espalhados pela planície de inundação AlenquerSantarém. As variaçôes espaciais dos constituintes da água mapeadas com a imagem hiperespectral do sensor Hyperion, forneceram informaçôes que podem ajudar a responder diversas questôes sobre as inter-relações das estruturas e funçōes que regem o ecossistema aquático amazônico durante o período de cheia na área de estudo.

Em geral, os algoritmos empíricos usados para estimativa da variação espacial das concentraçôes de COAs por meio de imagens de satélite são estabelecidos somente com auxílio de dados de verdade de campo e são úteis apenas dentro do contexto sazonal a partir do qual foi determinado. Na planície amazônica, isto ocorre devido às significativas alterações no intervalo de variaçãoo das concentrações dos COAs, impostas pelo pulso de inundação. Portanto, é preciso que os estudos avancem no sentido de

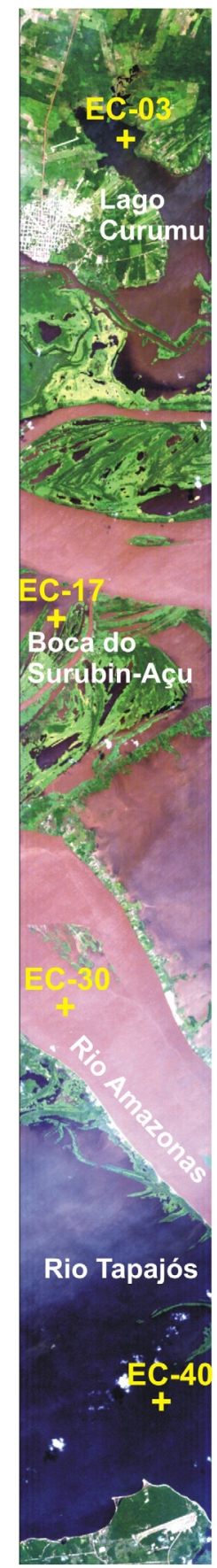

(a)

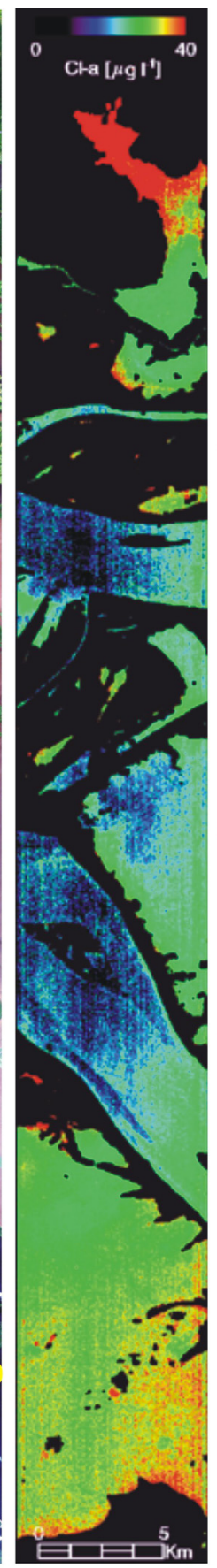

(b)

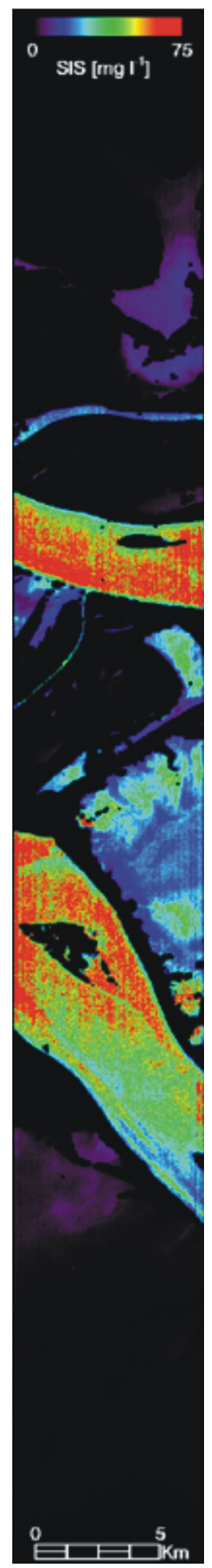

(c)
Figura 9 - (a) Imagem Hyperion/E0-1 adquirida no período de cheia (23/06/ 2005), em composição colorida normal: 640 nm (vermelho); 549 nm (verde); $457 \mathrm{~nm}$ (azul). Os pontos marcados com cruz amarela indicam os locais das estações de coleta EC-40, EC-30, EC-17 e EC-03. (b) Variações na concentração de clorofila $\left(\mathrm{Cl}-\mathrm{a}\right.$ em $\left.\mu \mathrm{g} \mathrm{l}^{-1}\right)$, estimadas a partir da $d R_{691 .}$ (c) Variações na concentração de sedimentos inorgânicos em suspensão (SIS em $\mathrm{mg} \cdot \mathrm{l}^{-1}$ ), estimadas a partir da $d R_{711}$. Em (b) e (c), os corpos d'água foram isolados com a aplicação de uma máscara (limiar de $1 \%$ de reflectância no SWIR). 
estabelecer outras regressões empíricas e modelos semi-analíticos, visando atender aos intervalos específicos de variação das concentraçôes dos COAs em cada período do pulso de inundação.

\section{AGRADECIMENTOS}

Os autores agradecem à NASA/LBA-Ecology pela aquisição da imagem e suporte financeiro, ao projeto GEOMA-MCT pelo financiamento parcial desta pesquisa e aos revisores anônimos pela contribuição de seus comentários. Conrado Rudorff agradece a CAPES pela bolsa de Mestrado em Sensoriamento Remoto no INPE, instituição onde foi realizada a pesquisa que deu origem a esse artigo. Evlyn Novo e Lênio Galvão agradecem ao CNPq pelo suporte financeiro.

\section{REFERÊNCIAS BIBLIOGRÁFICAS}

Adams, J.B.; Smith, M.O.; Johnson, P.E. 1986. Spectral mixture modeling: a new analysis of rock and soil types at the Viking Lander 1 site. Journal of Geophysical Research, 91: 8098-8112.

APHA. 1998. Standard Methods for the Examination of Water and Wastewater, 20. ed. American Public Health Association, Washington, DC.

Arst, H. 2003. Optical properties and remote sensing of multicomponental water bodies. Praxis Publishing Ltd, Chichester. 231pp.

Barbosa, C.C.F. 2005. Sensoriamento remoto da dinâmica de circulação da água do sistema planície de Curailrio Amazonas. Tese de doutorado, Instituto Nacional de Pesquisas Espaciais, São José dos Campos, São Paulo. 281pp.

Biggs, T.W.; Dunne, T.; Martinelli, L.A. 2004. Natural controls and human impacts on stream nutrient concentrations in a deforested region of the Brazilian Amazon basin. Biogeochemistry, 68(2): 227-257.

Bukata, R.P.J., J.H.; Kondratyev, K.Ya.; Pozdnyakov, D.V. 2000. Optical properties and remote sensing of inland and coastal waters. CRC Press LLC, Boca Raton. 362 pp.

Chen, Z.; Curran, P.J.; Hansom, J.D. 1992. Derivative reflectance spectroscopy to estimate suspended sediment concentration. Remote Sensing of Environment, 40(1): 67-77.

Curran, P.J.; Hansom, J.D.; Plummer, S.E.; Pedley, M.I. 1987. Multispectral remote-sensing of nearshore suspended sediments - A pilot study. International Journal of Remote Sensing, 8(1): 103-112.

Curran, P.J.; Novo, E.M.L.M. 1988. The relationship between suspended sediment concentration and remotely sensed spectral radiance: a review. Journal of Coastal Resources, 4: 351-368.

Dekker, A.G.; Malthus, T.J.; Seyhan, E. 1991. Quantitative modeling of inland water quality for high-resolution MSS systems. IEEE Transactions on Geoscience and Remote Sensing, 29(1): 89-95.

Fisher, T.R.; Parsley, P.E. 1979. Amazon lakes - water storage and nutrient stripping by algae. Limnology and Oceanography, 24(3): 547-553.

Forsberg, B.R.; Devol, A.H.; Richey, J.E.; Martinelli, L.A.; dos
Santos, H. 1988. Factors controlling nutrient concentrations in Amazon floodplain lakes. Limnology and Oceanography, 33(1): 41-56.

Furch, K.; Junk, W.J. 1997. Physicochemical conditions in the floodplains. In: Junk, W.J. (Ed). The Central Amazon Floodplain: Ecology of a Pulsing System. Springer-Verlag, Berlin. p. 69-108.

Gitelson, A. 1992. The peak near $700 \mathrm{~nm}$ on radiance spectra of algae and water - relationships of its magnitude and position with chlorophyll concentration. International Journal of Remote Sensing, 13(17): 3367-3373.

Goodenough, D.G.; Dyk, A.; Niemann, O.; Pearlman, J.S.; Chen, H.; Han, T.; Murdoch, M.; West, C. 2003. Processing Hyperion and ALI for forest classification. IEEE Transactions on Geoscience and Remote Sensing, 41(6): 1321-1331.

Han, L.; Rundquist, D.C. 1997. Comparison of NIR/RED ratio and first derivative of reflectance in estimating algal-chlorophyll concentration: a case study in a turbid reservoir. Remote Sensing of Environment, 62: 253-261.

Han, T.; Goodenough, D.G.; Dyk, A.; Love, J. 2002. Detection and correction of abnormal pixels in Hyperion images. In: Geoscience and Remote Sensing Symposium, 2002. Toronto, Canada. Proceedings... IEEE International, p. 1327-1330.

ImSpec. 2001. ACORN, MODTRAN, Boulder, CO, Analytical Imaging and Geophysics LLC.

Jensen, J.R. 2000. Remote sensing of water. In: Jensen, J.R. (Ed). Remote Sensing of the Environment: an Earth Resource Perspective. Prentice-Hall Inc., Upper Saddle River. p. 379-406.

Kaufman, Y.J. 1987. The effect of subpixel clouds on remote-sensing. International Journal of Remote Sensing, 8(6): 839-857.

Kaufman, Y.J. 1989. The atmospheric effect on remote sensing and its correction. In: Asrar, G. (Ed). Theory and Applications of Optical Remote Sensing. Wiley, New York. p. 336-428.

Kirk, J.T.O. 1994. Light and photosynthesis in aquatic ecosystems. 2a. ed. Cambridge University Press, Cambridge. 525pp.

Kondratyev, K.Y.; Pozdniakov, D.V. 1990. Passive and active optical remote-sensing of the inland water phytoplankton. ISPRS Journal of Photogrammetry and Remote Sensing, 44(5): 257-294.

Kruse, F.A. 2003. Preliminary results - hyperspectral mapping of coral reef systems using EO-1 Hyperion, Buck Island, U.S. Virgin Islands. In: JPL Airborne Earth Science Workshop, 12., 2003, Pasadena, USA. Proceedings... Pasadena: Jet Propulsion Laboratory, p. 157-173.

Méléder, V.; Barillé, L.; Launeau, P.; Carrère, V.; Rincé, Y. 2003. Spectrometric constraint in analysis of benthic diatom biomass using monospecific cultures. Remote Sensing of Environment, 88: 386-400.

Melack, J.M.; Forsberg, B.R. 2004. Biogeochemistry of Amazon floodplain lakes and associated wetlands. In: McClain, M.E.; Victoria, R.L.; Richey, J.E. (Eds). The Biogeochemistry of the Amazon Basin. Oxford University Press, New York. p. 235-274.

Mertes, L.A.K. 1994. Rates of floodplain sedimentation on the central Amazon River. Geology, 22(2): 171-174. 
Mertes, L.A.K.; Dunne, T.; Martinelli, L.A. 1996. Channelfloodplain geomorphology along the Solimoes-Amazon River, Brazil. Geological Society of America Bulletin, 108(9): 1089-1107.

Mertes, L.A.K.; Smith, M.O.; Adams, J.B. 1993. Estimating suspended sediment concentrations in surface waters of the Amazon River wetlands from Landsat images. Remote Sensing of Environment, 43(3): 281-301.

Milton, E. J. 1987. Principles of field spectroscopy. International Journal of Remote Sensing, 8(12): 1807-1827.

Neter, J.; Kutner, M.H.; Nachtsheim, C.J.; Wasserman, W. 1996. Applied linear statistical models. 4a. ed. McGraw-Hill, Irwin. 1388pp.

Novo, E.M.L.M.; Pereira Filho, W.; Melack, J.M. 2004. Assessing the utility of spectral band operators to reduce the influence of total suspended solids on the relationship between chlorophyll concentration and the bidirectional reflectance factor in Amazon waters. International Journal of Remote Sensing, 25(22): 51055116.

Novo, E.M.L.M.; Barbosa, C.C.F.; Freitas, R.M.; Shimabukuro, Y.E.; Melack, J.M.; Pereira Filho, W. 2006. Seasonal changes in chlorophyll distribution in Amazon floodplain lakes derived from MODIS images. Limnology, 7(3): 153-161.

Nush, E.A. 1980. Comparison of different methods for chlorophyll and phaeopigment determination. Archiv für Hydrobiologie Beiheft, 14: 14-39.

Pearlman, J.S.; Barry, P.S.; Segal, C.C.; Shepanski, J.; Beiso, D.; Carman, S.L. 2003. Hyperion, a space-based imaging spectrometer. IEEE Transactions on Geoscience and Remote Sensing, 41(6): 1160-1174.

Philpot, W.D. 1991. The derivative ratio algorithm: avoiding atmospheric effects in remote sensing. IEEE Transactions on Geoscience and Remote Sensing, 29: 250-357.

Rochelle-Newall, E.J.; Fisher, T.R. 2002. Chromophoric dissolved organic matter and dissolved organic carbon in Chesapeake Bay. Marine Chemistry, 77: 23-41.

Rudorff, C.M.; Novo, E.M.L.M.; Galvão, L.S. 2006. Spectral mixture analysis of inland tropical Amazon floodplain waters using EO-1 Hyperion. In: Geoscience and Remote Sensing Symposium 2006. Denver, USA. Proceedings... IEEE International.

Rudorff, C.M.; Novo, E.M.L.M.; Galvão, L.S. 2007. Spectral mixture analysis of EO-1 Hyperion imagery focused on the spatial-temporal variability of the Amazon floodplain multicomponental waters. In: Simpósio Brasileiro de Sensoriamento Remoto, 2007. Florianópolis, Brasil. Anais... Instituto Nacional de Pesquisas Espaciais.
Tsai, F.; Philpot, W. 1998. Derivative analysis of hyperspectral data. Remote Sensing of Environment, 66(1): 41-51.

Vittorio, E.B.; Dekker, A.G. 2003. Satellite hyperspectral remote sensing for estimating estuarine and costal water quality. IEEE Transactions on Geoscience and Remote Sensing, 41(6): 1378-1387.

Wetzel, R.G.; Likens, G.E. 1991. Limnological analyses. SpringerVerlag, New York. 391pp.

Recebido em 14/11/2006

Aceito em 18/05/2007

\section{LISTA DE ABREVIATURAS}

$\mathrm{CE}$ - condutividade elétrica

$\mathrm{Cl}-a-$ clorofila- $a$

$\mathrm{COA}$ - constituinte opticamente ativo

COD - carbono orgânico dissolvido

EC - estação de coleta

EO-1 - Earth Observing One

NIR - infravermelho próximo

OD - oxigênio dissolvido

PIAS - Planície de Inundação Alenquer-Santarém

SE-590 - Spectron Engineering 590

SIS - sedimentos inorgânicos em suspensão

SNR - razão sinal/ruído

SWIR - infravermelho de ondas curtas

VIS - visível

\section{LISTA DE SIMBOLOS}

$\lambda$-comprimento de onda

$R_{\lambda}$ - reflectância

$d R_{\lambda}$ - primeira derivada espectral 